\title{
Changing Trend of Antimicrobial Resistance in Acinetobacter baumannii
}

\author{
KZ MAMUN
}

Acinetobacter baumannii is an opportunistic nonfermentative, gram-negative, nonmotile, oxidasenegative bacillus. The natural reservoir still remains to be determined. However, it is found in many health care environments and is a very effective human colonizer in the hospital ${ }^{1}$. The combination of its environmental resilience and its wide range of resistance determinants renders it a successful nosocomial pathogen ${ }^{2} . A$. baumannii is mostly a cause of septicaemia, pneumonia and urinary tract infection following hospitalisation of patients with more severe illness. A. baumannii is frequently involved in numerous global outbreaks ${ }^{3}$, occurring mostly in intensive care units ${ }^{4}$.

The microorganisms that are mainly involved in antibiotic resistance are the so called ESKAPE pathogens, standing for Enterococcus faecium, Staphylococcus aureus, Klebsiella pneumoniae, Acinetobacter baumannii, Pseudomonas aeruginosa, and Enterobacteriaceae, capable of "escaping" from common antibacterial treatments ${ }^{3}$. Among these $A$. baumannii is one of the most challenging nosocomial pathogens due to its widespread emergence of antibiotic resistance.

In 1970 A baumannii was sensitive to most antibiotics, but now the pathogen appears to exhibit extensive resistance to most antibiotics ${ }^{5}$. The rapid emergence of multi- and pan drug-resistant strains of Acinetobacter highlights the organism's ability to quickly acclimatize to selective changes in environmental pressures. The up regulation of the organism's innate resistance mechanisms coupled with the acquisition of foreign determinants have played a crucial role in the express route the organism has taken to becoming a multidrugresistant pathogen ${ }^{6}$.

Acinetobacter spp. can acquire and spread antimicrobial resistance through conjugation of plasmids ${ }^{7,8}$. In addition Transposons (mobile genetic elements that are integrated into the chromosome or on plasmids) are also responsible in the dissemination of genetic determinants of resistance in Acinetobacter spp. ${ }^{9}{ }^{10}$. Many of these transposons contain integrons (predominantly class 1 ).
Integrons are genetic elements that, although unable to move themselves (they are carried either with plasmid or transposon), contain an int gene and gene cassettes that can be mobilized to other integrons or to secondary sites in the bacterial genome ${ }^{11,12,13}$. As in other gramnegative bacteria, an MDR phenotype in $A$. baumannii results when integron-borne resistance determinants against different classes of antibiotics coexist, giving rise to MDR gene cassettes. The selection and dissemination of the mobile elements carrying these resistance genes may be amplified in the clinical setting by the indiscriminate use of antibiotics ${ }^{14,15}$.

Fournier et al. ${ }^{16}$ identified an $86-\mathrm{kb}$ region called the AbaR1 resistance island in resistant strains of $A$. baumannii that contained a cluster of 45 resistance genes in the MDR isolates. Among the resistance genes determined were those coding for VEB-1, AmpC, and OXA-10 beta-lactamases, various aminoglycosidemodifying enzymes (AMEs), and tetracycline efflux pumps. Genetic analysis of AbaR1 demonstrated that it had similar mobile genetic elements and other genes previously identified in Pseudomonas spp., E coli, and Salmonella spp. However, the homologous location in the susceptible strain of $A$. baumannii consisted of a $20-\mathrm{kb}$ genomic island devoid of resistance genes.

Acinetobacter species are becoming a major cause of nosocomial infections and can present major challenges for physicians. Of growing concern is the increase in multidrug resistance exhibited by clinically relevant species ${ }^{17}$. Similar to western countries multidrugresistant isolates of $A$. baumannii have been reported increasingly during the last decade in Bangladesh, probably as a consequence of indiscriminate use of antimicrobial agents. A study in Bangladesh showed that antibiotic were prescribed on the basis of patient's complaints and also the antibiotics were used in inappropriate doses and durations (18).

A study in Bangladesh demonstrated that among $A$ baumannii, MBL encoding genes such as blaNDM-1 (83.33\%), blaVIM (66.67\%) and blaIMP (41.67\%); and ESBL encoding genes such as blaCTX-M-15 
(16.67\%) and blaOXA-1 (12.50\%) were detected by PCR (19). In another study in Dhaka Medical College, 6.37\% A baumannii were identified from 345 wound swab samples and of them $91 \%$ were multidrug resistant. All the identified $A$ baumannii were sensitive to colistin and $82 \%$ to imipenem. Out of 20 multidrug resistant $A$ baumannii, adeB gene was detected in 16(80\%). 4 (18\%) of 22 A baumannii were imipenem resistant. NDM-1 gene was detected in $2(50 \%)$ of the imipenem resistant strains of $A$ baumannii ${ }^{20}$.

In a recent study form Bangladesh on $A$ baumannii demonstrated that colistin resistance genes $p m r \mathrm{~A}$ (11.11\%), pmrB (28.89), pmrC (11.11\%), phoP (8.88\%), phoQ (4.44\%), mgrB (11.11\%), lpxA (28.89\%), lpxC (28.89), lpxD (28.89\%), mcr2 (8.88\%), mcr4 (4.44\%) and tigecycline resistance gene tet $A(8.88 \%)$ were detected by PCR using specific primers (21).

The present study was undertaken for identification and susceptibility on 69 isolates of Acinetobacter from critical care centers (CCC) from Combined Military Hospital (CMH). In the study, total 17 antibiotics were used to identify resistance pattern of Acinetobacter isolates. Ceftazidime (91.30\%), ceftriaxone (91.30\%), amikacin (52.20\%), gentamicin (33.33\%), cotrimoxazole (50\%) and ciprofloxacin (65.22\%) were mostly resistant. Only colistin appeared as most effective antibiotic with only $7.70 \%$ resistance rate.

Restriction of the use of antibiotics, especially those with broad-spectrum activity and those identified as antibiotics of last resort, is a necessary complement to any infection control strategy. The implementation of systems to monitor antimicrobial resistance and its relationship to antimicrobial use, as well as a program of antimicrobial stewardship, has been recommended by WHO. These are likely to have an impact on MDR $A$. baumannii, particularly as specific antimicrobials resistant to this organism were identified.

(J Bangladesh Coll Phys Surg 2020; 38: 56-58) DOI: https://doi.org/10.3329/jbcps.v38i2.46093

\section{KZMamun}

MBBS, MTrop Med (UK), Phd (UK), Post doctorate Fellow (UK)

Prof of Microbiology

Popular Medical College

\section{Reference:}

1. Bergogne-Bérézin E and Towner KJ. 1996. Acinetobacter spp. as nosocomial pathogens: microbiological, clinical, and epidemiological features. Clin Microbiol Rev. 9: 148-165

2. Nordmann, P. 2004. Acinetobacter baumannii, the nosocomial pathogen par excellence. Pathol. Biol. (Paris) 52:301-303.

3. Villegas MV, and Hartstein AI. 2003. Acinetobacter outbreaks, 1977-2000. Infect. Control Hosp. Epidemiol. 24:284-295.

4. Bergogne-Bérézin $\mathrm{E}$ and Towner KJ. 1996. Acinetobacter spp. as nosocomial pathogens: microbiological, clinical, and epidemiological features. Clin Microbiol Rev. 9: 148-165

5. Fournier PE, Vallenet D, Barbe V, Audic S, Ogata H, Poirel L, et. al., 2006. Comparative genomics of multidrug resistance in Acinetobacter baumannii. PLoS Genet. 2(1):e7.

6. Peleg AY, Seifert H, Paterson DL. 2008. Acinetobacter baumannii: emergence of a successful pathogen. Clin Microbiol Rev. ;21:538-82.

7. Goldstein, FW, Labigne-Roussel A, Gerbaud G, Carlier C, Collatz E, and Courvalin P. 1983. Transferable plasmidmediated antibiotic resistance in Acinetobacter. Plasmid 10:138-147.

8. Murray BE, and Moellering RC, Jr. 1980. Evidence of plasmid-mediated production of aminoglycoside-modifying enzymes not previously described in Acinetobacter. Antimicrob. Agents Chemother. 17:30-36.

9. Devaud M., Kayser FH, and Bachi B. 1982. Transposonmediated multiple antibiotic resistance in Acinetobacter strains. Antimicrob Agents Chemother. 22:323-329.

10. Palmen R., and Hellingwerf KJ. 1997. Uptake and processing of DNA by Acinetobacter calcoaceticus - a review. Gene 192:179-190.

11. Poirel LL. Cabanne H. Vahaboglu, and Nordmann P. 2005. Genetic environment and expression of the extendedspectrum -lactamase bla-PER-1 gene in gram-negative bacteria. Antimicrob Agents Chemother. 49:1708-1713.

12. Severino P, and Magalhaes VD. 2004. Integrons as tools for epidemiological studies. Clin Microbiol Infect. 10:156-162.

13. Turton, JF, Kaufmann ME, Glover J, Coelho JM, Warner M, Pike R, and Pitt TL. 2005. Detection and typing of integrons in epidemic strains of Acinetobacter baumannii found in the United Kingdom. J Clin Microbiol. 43: 3074-3082.

14. Seward, RJ. 1999. Detection of integrons in worldwide nosocomial isolates of Acinetobacter spp. Clin Microbiol. Infect. 5:308-318. 
15. Weldhagen, GF. 2004. Integrons and beta-lactamases - a novel perspective on resistance. Int. J Antimicrob Agents $23: 556-562$.

16. Fournier PE, Vallenet D, Barbe V, Audic S, Ogata, Poirel HL et. al., 2006. Comparative genomics of multidrug resistance in Acinetobacter baumannii. PLoS Genet. 2:e7.

17. Tabassum S. Multidrug-Resistant (MDR) - Acinetobacter: a Major Nosocomial Pathogen Challenging Physicians. 2007. Bangladesh J Med Microbiol. 01 (02): 65-68.

18. Mamun KZ, Shears P, Tabassum S, Hart CA. 1996. Antimicrobial use and antimicrobial resistance in rural Bangladesh. Trans Roy Soc Trop Med Hyg. 90:213

19. Bhuyan MM, Ritu S, Zubair AR, Surovi ES and Shamsuzzaman SM. 2020. In vitro and in vivo evaluation of antibiotic combination against imipenum resistant Acinetobacter baumannii strain isolated from Bangladeshi patients (Manuscript submitted in American Journal of Microbiological Research).

20. Hasan MJ and Shamsuzzaman SM. 2017. Distribution of adeB and NDM-1 genes in multidrug resistant Acinetobacter baumannii isolated from infected wound of patients admitted in a tertiary care hospital in Bangladesh. The Malaysian Journal of Pathology. 39(3):277-283.

21. Nazmun S. Development of heat inactivated whole cell vaccine against MDR Acinetobacter baumannii with detection of resistance genes among them. Personal communication (MPhil, thesis in Microbiology, Jan 2020). 